\title{
Neurobiologia do autismo infantil
}

\author{
Neurobiology of infantile autism \\ Neurobiología del autismo infantil
}

\section{Resumo}

Objetivo: discutir as características neurobiológicas do Transtorno do Espectro Autista (TEA) em crianças. Metodologia: foi realizada uma revisão narrativa com artigos dos últimos 10 anos (2010-2020), nas bases de dados PsycINFO, Medline, PubMed, SciELO, LILACS e Periódicos CAPES. Utilizaram-se como descritores "Autismo Infantil", "Transtorno Autista", "Infância", "Ciclo Vital", "Neurodesenvolvimento Infantil", "Neurobiologia", "Neurociências", "Neuroanatomia", "Neurofisiologia", "Transtorno do Espectro Autista" e o Booleano "AND". Todos estes materiais foram lidos na íntegra, categorizados e, posteriormente, analisados criticamente. Resultados e discussão: em indivíduos com TEA, há uma diminuição da conectividade entre o córtex pré-frontal medial e o córtex cingulado posterior; esta diminuição está relacionada a uma função social inferior quando comparada com crianças normais. Alteração semelhante foi encontrada em relação à rede frontoparietal relacionando-se aos comportamentos repetitivos observados no transtorno. Há, também, a hipótese relacionada a uma disfunção cerebelar e, mais especificamente, que a deleção da Proteína 1 do Complexo Esclerose Tuberosa (TSC1) - gene que codifica a proteína hamartina - nas células de Purkinje, reflita nos déficits sociais e comportamentais em indivíduos com TEA. Além disso, é sugerido que o fenótipo do transtorno esteja associado a uma perturbação nos circuitos que conectam o cerebelo ao córtex pré-frontal medial e, também, a uma maior ativação desta última região. Conclusão: ainda não se tem uma conclusão satisfatória e patognomônica da neurobiologia do TEA em crianças; as alterações neurofuncionais mais comuns no TEA estão relacionadas às redes cerebelares e às Células de Purkinje.

Palavras-chave: Neurobiologia; Transtorno autístico; Transtornos do neurodesenvolvimento; Transtorno do espectro autista.

\begin{abstract}
Objective: to discuss the neurobiological characteristics of Autism Spectrum Disorder (ASD) in children. Methodology: a narrative review was conducted based on articles from the last 10 years (2010-2020) published on the databases PsycINFO, Medline, PubMed, SciELO, LILACS and Periódicos CAPES. The descriptors used are "Infantile Autism", “Autism Disorder", "Childhood", "Vital Cycle", "Child Neurodevelopment", "Neurobiology", "Neuroscience", "Neuroanatomy", "Autism Spectrum Disorder" and the Boolean "AND". The material was entirely read, categorized, and subsequently, critically analyzed. Results and discussion: among ASD individuals there is a decrease in connectivity between the medial prefrontal cortex and the posterior cingulate cortex; this decrease is related to a reduced social function when compared to healthy children. Similar alteration was found regarding the frontoparietal network which related to the repetitive behaviors observed in the disorder. There also is a hypothesis concerning cerebellar dysfunction and more specifically, that the Tuberous Sclerosis Complex 1 (TSC 1) deletion gene that encodes the hamartin protein - in the Purkinje cells reflects on the social and behavioral deficits among ASD individuals. Furthermore, it is suggested that the disorder phenotype is associated with a disturbance in the networks that connect the cerebellum to the medial prefrontal cortex also due to a higher activation in the last region. Conclusion: there still is no satisfactory and pathognomonic conclusion regarding the neurobiology of ASD in children. The most common neurofunctional alterations in ASD are related to cerebellar networks and Purkinje cells. Keywords: Neurobiology; Autistic disorder; Neurodevelopmental disorders; Autism spectrum disorder.
\end{abstract}

\section{Resumen}

Objetivo: discutir las características neurobiológicas del Trastorno del Espectro Autista (TEA) en niños. Metodología: se realizó una revisión narrativa con artículos de los últimos 10 años (2010-2020), en las bases de datos PsycINFO, 
Medline, PubMed, SciELO, LILACS y CAPES. Se utilizaron los descriptores "Autismo infantil", "Trastorno autista", "Infancia", "Ciclo de vida", "Neurodesarrollo infantil", "Neurobiología", "Neurociencia", "Neuroanatomía", "Neurofisiología", "Trastorno del Espectro Autista" y el booleano "Y". Todos estos materiales fueron leídos íntegramente, categorizados y analizados críticamente. Resultados y discusión: en individuos con TEA, hay una disminución en la conectividad entre la corteza prefrontal medial y la corteza cingulada posterior; esta disminución está relacionada con una función social más baja en comparación con los niños normales. Se encontró un cambio similar con relación a la red frontoparietal, en relación con las conductas repetitivas observadas en el trastorno. También existe la hipótesis relacionada con una disfunción cerebelosa y, más específicamente, que la deleción de Proteína 1 del Complejo de la Esclerosis Tuberosa (TSC1), un gen que codifica la proteína hamartina, en las células de Purkinje, refleja los déficits sociales y del comportamiento en los individuos con TEA. Además, se sugiere que el fenotipo del trastorno se asocia con una alteración en los circuitos que conectan el cerebelo con la corteza prefrontal medial y también con una mayor activación de esta última región. Conclusión: aún no existe una conclusión satisfactoria y patognomónica sobre neurobiología del TEA en niños; los cambios neurofuncionales más comunes están relacionados con las redes cerebelosas y las células de Purkinje.

Palabras clave: Neurobiología; Transtorno autístico; Trastornos del neurodesarrollo; Trastorno del espectro autista.

\section{Introduçãa}

Inicialmente, o termo "Autismo" foi cunhado pelo psiquiatra suíço Eugen Bleuler e atribuído ao afastamento da realidade, observado em pacientes com esquizofrenia (Crespi, 2010). Entretanto, em 1943, ao acompanhar uma coorte de onze crianças com comportamentos semelhantes em Maryland - EUA, Leo Kanner ressignificou o termo "Autismo" e introduziu o conceito de "Autismo Infantil". Em seu artigo, denominado "Distúrbio Autístico do Contato Afetivo" (Kanner, 1943), descreveu cada caso de forma minuciosa, baseando-se em suas próprias observações e no relato dos pais das crianças, as quais, de acordo com o psiquiatra, não possuíam a habilidade inata de estabelecer contato com outras pessoas (Harris, 2018).

Na década de 1970, foi unânime o reconhecimento da importância de se estudar o autismo de maneira individualizada, e não mais associado à esquizofrenia, além de sistematizar um modelo diagnóstico para o transtorno. Entre os autores que contribuíram com o desenvolvimento das pesquisas relacionadas ao Autismo Infantil após a publicação de Kanner, destaca-se Michael Rutter, professor da King's College, em Londres (Volkmar \& McPartland, 2014). Finalmente, em 1980, o diagnóstico de Autismo Infantil foi reconhecido pelo Diagnóstico de Transtornos Mentais III (DSM-II).

Atualmente, o Autismo Infantil está incluído no Código Internacional de Doenças (CID-10) na categoria F84, que aborda os Transtornos Globais do Desenvolvimento, e na subcategoria F84.0. Nesta subcategoria estão excluídos o Autismo Atípico e Síndrome de Asperger. Em contrapartida, o DSM-V, engloba todos estes diagnósticos em um único transtorno, denominado Transtorno do Espectro Autista (TEA), caracterizado por déficits na comunicação e interação social e por padrões de comportamento restritivos e repetitivos, e que devem estar presentes precocemente no desenvolvimento (APA, 2013). Esta mesma classificação - utilizada pelo DSM-V - será adotada pelo novo Código Internacional de Doenças (CID-11), que entrará em vigor em janeiro de 2022. Desse modo, o Transtorno do Espectro Autista será incluído na categoria 6A02, cujas subcategorias estão relacionadas à presença ou à ausência de deficiência intelectual e comprometimento da linguagem funcional.

Tendo em vista que o Autismo Infantil é descrito como um transtorno multissistêmico, o presente estudo consiste em uma revisão bibliográfica narrativa de literatura, que tem por finalidade reunir e destacar as principais características neurobiológicas observadas nesta patologia.

\section{Metodologia}

O presente estudo, de natureza qualitativa e descritiva, trata-se de uma revisão bibliográfica da literatura, do tipo narrativa. Este tipo de revisão também é conhecido como "tradicional", e permite ao leitor a atualização acerca de fatos e 
argumentos já existentes, fomentando a compreensão e estimulando pensamentos controversos (Chazan, Fortes \& Camargo, 2020). Embora a força de evidência científica da revisão bibliográfica narrativa seja considerada baixa devido à impossibilidade de reprodução de sua metodologia, a escolha se deu ao fato de constituir um tipo de publicação ampla e apropriada para descrever e discutir o desenvolvimento de um tema. Ademais, sabe-se que proporciona o debate sobre certos assuntos, levantando questionamentos e corroborando o conhecimento em curto espaço de tempo (Rother, 2007). Vale ressaltar que, de acordo com Depolito et al. (2020), a revisão do tipo narrativa é de fundamental importância para divulgar uma análise abrangente e reflexiva acerca do tema que se pretende explorar.

À vista disso, a pesquisa foi realizada utilizando-se artigos indexados nas bases de dados PsycINFO, Medline, PubMed, Biblioteca Eletrônica Científica Online - SciELO, Literatura Latino-Americana e do Caribe em Ciências da Saúde LILACS e Periódicos CAPES. Os descritores utilizados na busca foram: "Autismo Infantil”, "Transtorno Autista", "Infância", "Ciclo Vital", "Neurodesenvolvimento Infantil", "Neurobiologia", "Neurociências", "Neuroanatomia", "Neurofisiologia", "Transtorno do Espectro Autista" e o Booleano "AND". Os critérios de inclusão foram: a) artigos publicados nos últimos 10 anos (2010-2020); b) artigos que possuíam no título e/ou resumo os descritores citados anteriormente.

Para a concretização do objetivo, foi realizada uma leitura dos resumos de cada artigo e, posteriormente, foram selecionados apenas aqueles que poderiam contribuir com a discussão do tema. Após esta fase, os materiais foram lidos na íntegra, categorizados de acordo com os tópicos apresentados no decorrer do presente artigo e, sucessivamente, analisados criticamente, a fim de extrair as informações essenciais. Em seguida, procedeu-se à discussão, comparando-se os resultados de cada artigo selecionado.

\section{Resultados e Discussão}

\subsection{Desenvolvimento neuropsicomotor - padrões de normalidade}

De acordo com Moraes, Weber, Santos e Almeida (2010), o desenvolvimento infantil deve ser entendido como um processo multifatorial, e que abrange as esferas cognitiva, social e afetiva, além do crescimento físico em si. Dentre os fatores predisponentes associados a um desvio dos padrões de normalidade no desenvolvimento neuropsicomotor infantil, destaca-se a condição socioeconômica em que se insere a criança. Para o acompanhamento de indivíduos de 0-6 anos de idade, foi criado o Teste de Triagem de Desenvolvimento de Denver (TTDD), que avalia o desempenho da criança por meio de 125 itens, divididos em 4 áreas (pessoal-social, motricidade fina, motricidade ampla e linguagem), a partir da observação da execução dos itens e do relato dos pais (Custódio, Crepaldi \& Cruz, 2012).

Vale ressaltar que o TTDD serve apenas como instrumento de triagem e rastreamento de possíveis desvios no desenvolvimento infantil; entretanto, para o diagnóstico clínico, são necessárias avaliações complementares (Brito, Vieira, Costa \& Oliveira 2011). Diante do exposto, e em virtude da diversidade etiológica referente aos déficits no crescimento e desenvolvimento infantil, faz-se necessário o acompanhamento continuado, que compreende o período pré-natal e pós-natal, com atenção especial às vulnerabilidades e, por conseguinte, a promoção de assistência aos possíveis agravos de saúde da criança.

\subsection{Default Mode Network (DMN)}

A Default Mode Network diz respeito a uma rede de áreas cerebrais em que se observa um declínio da atividade durante tarefas que demandem atenção, e compreende três estruturas principais: córtex pré-frontal ventromedial, o córtex préfrontal dorsomedial e o córtex cingulado posterior e pré-cuneo adjacente, além do córtex parietal lateral (Raichle, 2015). A 
associação entre essas regiões se faz possível por meio do uso de instrumentos de quantificação das respostas dependentes do nível de oxigenação sanguínea (Blood Oxygen Level Dependent - BOLD), como a Ressonância Magnética Funcional em Estado de Repouso (fMRI). Embora mais evidências sejam necessárias, sugere-se que a conectividade funcional entre as áreas que compõem a rede está diminuída em crianças, seja devido à imaturidade da formação desta rede ou à maior movimentação destes participantes durante a avaliação (Mak et al., 2017). Raichle (2015) salienta que o córtex pré-frontal ventromedial é um elemento importante em uma rede de áreas que estão associadas ao comportamento social e à personalidade. Além disso, o estado emocional do indivíduo reflete diretamente o nível de atividade observado nesta estrutura. Por outro lado, é referida uma associação do córtex pré-frontal dorsomedial ao processamento individual das informações e, consequentemente, ao julgamento. A última estrutura, o córtex cingulado posterior, em associação ao pré-cuneo medial, está relacionada à recordação de experiências anteriores.

À vista disso, Yerys et al. (2015), ao analisarem vinte e duas crianças diagnosticadas com Transtorno do Espectro Autista por meio de imagens BOLD, demonstraram que há uma diminuição da conectividade entre o córtex pré-frontal medial e o córtex cingulado posterior, e que esta diminuição está relacionada a uma função social inferior quando comparada ao grupo controle. Além disso, observou-se uma interação maior entre a DMN e outras redes, responsáveis pelas respostas motoras, por exemplo. Alteração semelhante foi encontrada em relação à rede frontoparietal relacionando-se aos comportamentos repetitivos observados no TEA. Ademais, Assaf et al. (2010) reiteram o achado citado anteriormente, de que há uma diminuição da conectividade entre o córtex pré-frontal medial/córtex cingulado anterior e a região do pré-cuneo, e que a força desta conectividade está negativamente relacionada aos déficits sociais e de comunicação observados nos indivíduos com TEA. Por fim, He et al. (2018), evidenciaram uma menor variância na Dynamic Functional Conectivity (DFC) - isto é, alterações na conectividade funcional em um período de tempo - entre as regiões da DMN, como o córtex cingulado posterior, e o giro pré-central direito em crianças diagnosticadas com TEA, e este achado pode estar associado aos déficits no âmbito cognitivo-social. Além disso, sugere-se que uma maior diminuição da variância na DFC resultaria em déficits mais severos. Outras evidências, como variações nos padrões dinâmicos de conectividade funcional entre crianças diagnosticadas com TEA e controles pareados por idade, poderiam contribuir com a neuropatologia do transtorno em questão.

\subsection{Alterações Estruturais}

Ao relacionar os déficits de fala às lesões encontradas em estudos post mortem realizados no encéfalo de seus pacientes, o médico francês Pierre Paul Broca (1824-1880) impulsionou os estudos que tinham como finalidade entender as repercussões da assimetria cerebral, característica imprescindível para o funcionamento sensorial, cognitivo e motor (Güntürkün, Ströckens \& Ocklenburg, 2019). Floris e Howells (2018) reúnem diversos autores que ratificam a descoberta de Broca e acrescentam que as diferenças são compreendidas não apenas no âmbito funcional, mas também no anatômico. Além disso, sabe-se que essas diferenças entre hemisférios são resultantes da expressão assimétrica de genes durante a gestação e, ainda, que tendem a aumentar em decorrência do envelhecimento. Em outro estudo, Floris et al. (2016) indicam que há uma alteração atípica na assimetria em indivíduos com TEA, e que esta alteração está associada a funções motoras. Ademais, mediante análise pelo método Morfometria Baseada em Voxel (Voxel Based Morphometry - VBM), Yang et al. (2018) apontam uma diminuição da substância cinzenta em crianças diagnosticadas com TEA em comparação aos controles, e esta é predominante na região esquerda do cerebelo. Outrossim, também foi descrito um comprometimento na integridade da substância branca, principalmente nas regiões do giro frontal, do lóbulo parietal e da Área Motora Suplementar direita. Por fim, ao comparar crianças diagnosticadas com TEA e crianças com desenvolvimento neuropsicomotor normal por meio de 
Imagens de Difusão por Ressonância Magnética (IRM de difusão), Vogan et al. (2016), descreveram uma redução da anisotropia (Fractional Anisotropy - FA) e da difusividade axial (Axial Diffusivity - AD), revelando um extenso comprometimento da substância branca entre o grupo com TEA. De acordo com os autores, esses achados são compatíveis com estudos anteriores, que também demonstraram uma redução da substância branca, principalmente na região do corpo caloso, que persiste desde a infância até a vida adulta.

\subsection{Disfunções Cerebelares}

A despeito de terem sido realizadas em modelos animais, pesquisas sugerem que o Transtorno do Espectro Autista está relacionado a uma disfunção cerebelar e, mais especificamente, que a deleção da Proteína 1 do Complexo Esclerose Tuberosa (TSC1) - gene que codifica a proteína hamartina - nas células de Purkinje reflete nos déficits sociais e no comportamento repetitivo observado em indivíduos com TEA. Além disso, é sugerido que o fenótipo do transtorno esteja associado a uma perturbação nos circuitos que conectam o cerebelo ao córtex pré-frontal medial e, também, a uma maior ativação desta última região (Kelly et al., 2020). Outrossim, D’Mello e Stoodley (2015) relatam que lesões no cerebelo devido a hemorragias, por exemplo - são consideradas fator de risco para o desenvolvimento de TEA; e sabe-se que em crianças prematuras, nas quais há uma interrupção do desenvolvimento cerebelar - e uma intervenção na maturação das estruturas ou dos circuitos - há um aumento neste risco. As autoras ainda sugerem, com base em estudos anteriores, que alterações quantitativas e estruturais nas fibras cerebelares, bem como uma diminuição no número e no tamanho das células de Purkinje também estão envolvidos na patogenia do transtorno. Estudos post mortem realizados em indivíduos diagnosticados com TEA ratificam as alterações nas células de Purkinje descritas anteriormente. Especula-se que estas perdas ocorram nos estágios iniciais do desenvolvimento (Bruchhage, Bucci \& Becker, 2018). Outros estudos também sugerem que a hipoplasia do vermis cerebelar está relacionada à disfunção cognitiva em crianças com autismo (Fatemi et al., 2012) e que lesões ou malformações nesta região estão associadas a comportamentos que se assemelham aos observados no transtorno (Hampson \& Blatt, 2015).

\section{Conclusão}

Embora exista um número significativo de estudos que têm por finalidade elucidar os aspectos neurobiológicos de indivíduos portadores do Transtorno do Espectro Autista, ainda não é possível estabelecer uma característica patognomônica que esteja associada ao Autismo Infantil, visto que várias alterações estão presentes em outros transtornos neurocognitivos. Até o presente momento, as disfunções nos circuitos cerebelares e as modificações quantitativas e qualitativas referente às células de Purkinje são amplamente relatadas na literatura e estão relacionadas à patogenia do TEA. Entretanto, tais alterações não estão presentes exclusivamente em crianças. Ademais, sabe-se que a prematuridade pode ser considerada fator de risco para o desenvolvimento de TEA pela possibilidade de interrupção do desenvolvimento cerebelar. Por fim, também são descritas alterações na Default Mode Network (DMN) em crianças portadoras de TEA, e estas compreendem uma diminuição na conectividade funcional entre as áreas que compõem a rede.

À vista disso, observa-se a necessidade da realização de futuros estudos que possam vir a elucidar as alterações neurobiológicas relacionadas ao Autismo Infantil. Para isso, os autores pretendem estender os estudos na área por meio de comparações entre a neurobiologia do autismo com outros transtornos neuropsiquiátricos infantis e, dessa forma, compreender quais são os padrões mais característicos do autismo infantil em detrimento de outros transtornos. Por conseguinte, tais estudos 
podem vir a colaborar com o esclarecimento da etiopatogenia e com o estabelecimento de uma terapêutica cada vez mais específica e eficaz para o transtorno em questão.

\section{Referências}

Assaf, M., Jagannathan, K., Calhoun, V. D., Miller, L., Stevens, M. C., Sahl, R., Pearlson, G. D. (2010). Abnormal functional connectivity of default mode sub-networks in autism spectrum disorder patients. NeuroImage, 53(1), 247-256. 10.1016/j.neuroimage.2010.05.067

Brito, C. M., Vieira, G. O., Costa, M. D., \& Oliveira, N. F. (2011). Desenvolvimento neuropsicomotor: O teste de Denver na triagem dos atrasos cognitivos e neuromotores de pré-escolares. Cadernos De Saúde Pública, 27(7), 1403-1414. 10.1590/s0102-311x2011000700015

Bruchhage, M. M., Bucci, M., \& Becker, E. B. (2018). Cerebellar involvement in autism and ADHD. The Cerebellum: Disorders and Treatment Handbook of Clinical Neurology, 61-72. 10.1016/b978-0-444-64189-2.00004-4

Chazan, L. F., Fortes, S. L. C. L., \& Camargo Junior, K. R. D. (2020). Apoio Matricial em Saúde Mental: revisão narrativa do uso dos conceitos horizontalidade e supervisão e suas implicações nas práticas. Ciência \& Saúde Coletiva, 25, 3251-3260. https://doi.org/10.1590/1413-81232020258.31942018

Crespi, B. J. (2010). Revisiting Bleuler: Relationship between autism and schizophrenia. British Journal of Psychiatry, 196(6), 495-495. doi:10.1192/bjp.196.6.495

Custódio, Z. A., Crepaldi, M. A., \& Cruz, R. M. (2012). Desenvolvimento de crianças nascidas pré-termo avaliado pelo teste de Denver-II: Revisão da produção científica brasileira. Psicologia: Reflexão E Crítica, 25(2), 400-406. 10.1590/s0102-79722012000200022

Depolito, S. C. P., Moraes, L. L., Siqueira, W. G., Sales Baquião, L. M., Januário, G. C., \& Morceli, G. (2020). Atuação da equipe de enfermagem frente ao desmame precoce: Uma revisão narrativa. Saúde Coletiva (Barueri), 10(55), 2915-2924. https://doi.org/10.36489/saudecoletiva.2020v10i55p2915-2924

D'mello, A. M., \& Stoodley, C. J. (2015). Cerebro-cerebellar circuits in autism spectrum disorder. Frontiers in Neuroscience, 9. 10.3389/fnins.2015.00408

Fatemi, S. H., Aldinger, K. A., Ashwood, P., Bauman, M. L., Blaha, C. D., Blatt, G. J., Welsh, J. P. (2012). Consensus Paper: Pathological Role of the Cerebellum in Autism. The Cerebellum, 11(3), 777-807. 10.1007/s12311-012-0355-9

Floris, D. L., \& Howells, H. (2018). Atypical structural and functional motor networks in autism. Progress in Brain Research Cerebral Lateralization and Cognition: Evolutionary and Developmental Investigations of Behavioral Biases, 207-248. 10.1016/bs.pbr.2018.06.010

Floris, D. L., Barber, A. D., Nebel, M. B., Martinelli, M., Lai, M., Crocetti, D., Mostofsky, S. H. (2016). Atypical lateralization of motor circuit functional connectivity in children with autism is associated with motor deficits. Molecular Autism, 7(1). 10.1186/s13229-016-0096-6

Güntürkün, O., Ströckens, F., \& Ocklenburg, S. (2020). Brain Lateralization: A Comparative Perspective. Physiological Reviews, 100(3), 1019-1063. 10.1152/physrev.00006.2019

Hampson, D. R., \& Blatt, G. J. (2015). Autism spectrum disorders and neuropathology of the cerebellum. Frontiers in Neuroscience, 9. $10.3389 /$ fnins.2015.00420

Harris, J. (2018). Leo Kanner and autism: A 75-year perspective. International Review of Psychiatry, 30(1), 3-17. 10.1080/09540261.2018.1455646

He, C., Chen, Y., Jian, T., Chen, H., Guo, X., Wang, J., Duan, X. (2018). Dynamic functional connectivity analysis reveals de creased variability of the defaultmode network in developing autistic brain. Autism Research, 11(11), 1479-1493. 10.1002/aur.2020

Kelly, E., Meng, F., Fujita, H., Morgado, F., Kazemi, Y., Rice, L. C., Tsai, P. T. (2020). Regulation of autism-relevant behaviors by cerebellar-prefrontal cortical circuits. Nature Neuroscience, 23(9), 1102-1110. 10.1038/s41593-020-0665-z

Mak, L. E., Minuzzi, L., Macqueen, G., Hall, G., Kennedy, S. H., \& Milev, R. (2017). The Default Mode Network in Healthy Individuals: A Systematic Review and Meta-Analysis. Brain Connectivity, 7(1), 25-33. 10.1089/brain.2016.0438

Moraes, M. W., Weber, A. P., Marcela De Castro E Oliveira Santos, \& Almeida, F. D. (2010). Denver II: Evaluation of the development of children treated in the outpatient clinic of Project Einstein in the Community of Paraisópolis. Einstein (São Paulo), 8(2), 149-153. 10.1590/s1679-45082010ao1620

Raichle, M. E. (2015). The Brain's Default Mode Network. Annual Review of Neuroscience, 38(1), 433-447. 10.1146/annurev-neuro-071013-014030

Rother, E. T. (2007). Revisão sistemática X revisão narrativa. Acta Paulista De Enfermagem, 20(2), V-Vi. 10.1590/s0103-21002007000200001

Vogan, V. M., Morgan, B. R., Leung, R. C., Anagnostou, E., Doyle-Thomas, K., \& Taylor, M. J. (2016). Widespread White Matter Differences in Children and Adolescents with Autism Spectrum Disorder. Journal of Autism and Developmental Disorders, 46(6), 2138-2147. 10.1007/s 10803-016-2744-2

Volkmar, F. R., \& Mcpartland, J. C. (2014). From Kanner to DSM-5: Autism as an Evolving Diagnostic Concept. Annual Review of Clinical Psychology, 10(1), 193-212. 10.1146/annurev-clinpsy-032813-153710

Yang, Q., Huang, P., Li, C., Fang, P., Zhao, N., Nan, J., Cui, L. (2018). Mapping alterations of gray matter volume and white matter integrity in children with autism spectrum disorder. NeuroReport, 29(14), 1188-1192. 10.1097/wnr.0000000000001094 
Research, Society and Development, v. 10, n. 1, e11910111495, 2021 (CC BY 4.0) | ISSN 2525-3409 | DOI: http://dx.doi.org/10.33448/rsd-v10i1.11495

Yerys, B. E., Gordon, E. M., Abrams, D. N., Satterthwaite, T. D., Weinblatt, R., Jankowski, K. F., Vaidya, C. J. (2015). Default mode network segregation and social deficits in autism spectrum disorder: Evidence from non-medicated children. NeuroImage: Clinical, 9, 223-232. 10.1016/j.nicl.2015.07.018 